\title{
Structure and Scm3-mediated assembly of budding yeast centromeric nucleosomes
}

\author{
Mekonnen Lemma Dechassa', Katharina Wyns', Ming Li ${ }^{2}$, Michael A. Hall ${ }^{3}$, Michelle D. Wang $2,3,4$ \\ $\&$ Karolin Luger ${ }^{1,4}$
}

Much controversy exists regarding the structural organization of the yeast centromeric nucleosome and the role of the nonhistone protein, $\mathrm{Scm} 3$, in its assembly and architecture. Here we show that the substitution of $\mathrm{H} 3$ with its centromeric variant $\mathrm{Cse} 4$ results in octameric nucleosomes that organize DNA in a left-handed superhelix. We demonstrate by singlemolecule approaches, micrococcal nuclease digestion and small-angle $\mathrm{X}$-ray scattering that Cse4-nucleosomes exhibit an open conformation with weakly bound terminal DNA segments. The Cse4-octamer does not preferentially form nucleosomes on its cognate centromeric DNA. We show that $\mathrm{Scm} 3$ functions as a Cse4-specific nucleosome assembly factor, and that the resulting octameric nucleosomes do not contain $\mathrm{Scm} 3$ as a stably bound component. Taken together, our data provide insights into the assembly and structural features of the budding yeast centromeric nucleosome.

\footnotetext{
1 Department of Biochemistry and Molecular Biology, Colorado State University, Fort Collins, Colorado 80523, USA. ${ }^{2}$ Department of Chemistry and Chemical Biology, Cornell University, Ithaca, New York 14853, USA. ${ }^{3}$ Department of Physics, Cornell University, Ithaca, New York 14853 , USA. ${ }^{4}$ Howard Hughes Medical Institute, 4000 Jones Bridge Road, Chevy Chase, MD 20815-6789, USA . Correspondence and requests for materials should be addressed to K.L. (email: kluger@lamar.colostate.edu).
} 
T he centromere is a specific locus on each chromosome that directs the formation of the kinetochore, a multiprotein complex that interacts with spindle microtubules to promote proper chromosomal alignment and segregation during cell division ${ }^{1}$. The replacement of canonical (major-type) H3 with the centromere-specific histone variant CenH3 (known as CENP-A in humans and Cse4 in budding yeast) is essential for centromere function. CenH3 proteins from all organisms have a $\sim 90$ aminoacid carboxy-terminal histone domain that exhibits $\sim 60 \%$ aminoacid sequence identity with canonical H3 (Supplementary Fig. S1a). Although canonical $\mathrm{H} 3$ is highly conserved between species, CenH3 is evolving rapidly ${ }^{2}$. The histone domain exhibits only $45-50 \%$ identity between various species, and its amino (N)-terminal tail is even more divergent in both length and amino-acid composition ${ }^{3}$.

The protein composition, stoichiometry and structure of centromeric nucleosomes remain controversial. Mammalian centromeric nucleosomes are composed of two copies of each histone (CENP$\mathrm{A} / \mathrm{H} 4 / \mathrm{H} 2 \mathrm{~A} / \mathrm{H} 2 \mathrm{~B})_{2}$ (refs 4-6). Drosophila centromeric nucleosomes have been described as 'hemisomes' in vivo, containing only a single copy of each histone $(\mathrm{CenH} 3 / \mathrm{H} 4 / \mathrm{H} 2 \mathrm{~A} / \mathrm{H} 2 \mathrm{~B})^{7}$. In contrast, budding yeast centromeric nucleosomes are thought to form either octameric or hexameric complexes ${ }^{8-10}$. In the proposed hexameric structure, the two $\mathrm{H} 2 \mathrm{~A} / \mathrm{H} 2 \mathrm{~B}$ dimers are replaced by the nonhistone protein $\mathrm{Scm} 3$ (ref. 11). In fission yeast, $\mathrm{H} 2 \mathrm{~A} / \mathrm{H} 2 \mathrm{~B}$ dimers are depleted from the inner centromere regions containing CenH3 (ref. 12). Reports on the conformation of DNA in centromeric nucleosomes are also contradictory ${ }^{5,13}$. There is evidence from in vitro (Drosophila) and in vivo (Saccharomyces cerevisiae) experiments that the assembly of centromeric nucleosomes onto plasmid DNA induces positive supercoils ${ }^{13}$. This suggests that the DNA in centromeric nucleosomes is wrapped around the histone complex in a right-handed, as opposed to the canonical left-handed, superhelix. Because of obvious structural constraints ${ }^{14}$, the composition of these particles might be tetrameric or hexameric ${ }^{13}$, suggesting a third type of yeast centromeric 'nucleosome'.

Structural and in vivo studies of human CENP-A show that the 22 amino-acid CENP-A-targeting domain is critical for its localization to the centromere ${ }^{15}$. The structure of the (CENP-A/H4) tetramer-in the absence of DNA - revealed a high degree of similarity to major-type $\mathrm{H} 3 / \mathrm{H} 4$ in a nucleosome. However, several unique features were noted ${ }^{5}$. As only two of the eight amino acids responsible for the unique structural features of the (CENP-A/H4) tetramer are conserved in Cse4 (Supplementary Fig. S1a), the interpretations derived from the human (CENP-A/H4) $)_{2}$ structure cannot easily be applied to the yeast (Cse $4 / \mathrm{H} 4)_{2}$ tetramer.

Budding yeast centromeres consist of a single $125 \mathrm{bp}$ consensus sequence that is strictly required for centromere formation and that harbours a single Cse4-containing nucleosome $e^{16,17}$. The CEN regions of all 16 yeast centromeres all contain three consensus elements, termed CDE I, CDE II and CDE III ${ }^{18}$. The site of Cse 4 occupancy is CDE II, a highly AT-rich (>90\%) 78-86 bp DNA region ${ }^{19}$. CEN DNA-binding factor 3 (CBF3), binds to the CDE III consensus element, and the CDE III-CBF3 complex is required for centromere chromatin formation and function ${ }^{20}$. CDE I is the binding site for Cbf1 protein, which sharply bend the CEN DNA ${ }^{18}$. The interaction of CBF3 and Cbf1 with nucleosomal DNA may require special, as yet unidentified structural features of the Cse4-nucleosome. Moreover, the effect of this unusual DNA sequence on the structure, composition and stability of the centromeric yeast nucleosome has not been investigated.

There is evidence that CenH3-specific factors deposit CenH3 at the appropriate location. In budding and fission yeast, this role is played by $\mathrm{Scm} 3$, a protein that is required for $\mathrm{G} 2 / \mathrm{M}$ progression and incorporation of CenH3 to the centromere ${ }^{8,11,12,21,22}$. The functional mammalian homologue HJURP also has histone chaperone-like activity and facilitates deposition of the (CENP-A-H4) 2 tetramer onto $\mathrm{DNA}^{23-25}$. In budding yeast, $\mathrm{Scm} 3$ interacts directly with the $\mathrm{Ndc10}$ subunit of the CBF3 complex ${ }^{26}$ and depends on $\mathrm{Ndc10}$ for its association with the centromere ${ }^{21}$. In vitro, $\mathrm{Scm} 3$ forms a stable complex with Cse4/H4 (refs 8, 11) and displaces H2A/H2B dimers from refolded histone octamer ${ }^{11}$; however, whether or not $\mathrm{Scm} 3$ is a permanent component of the centromeric 'nucleosome' remains controversial $^{8,11}$.

Here we present biochemical and biophysical studies that reveal structural features of budding yeast Cse4-nucleosomes and shed light on the function of $\mathrm{Scm} 3$ in nucleosome assembly. We find that the Cse4-nucleosome is composed of two copies each of Cse4, $\mathrm{H} 4, \mathrm{H} 2 \mathrm{~A}$ and H2B that organize DNA in a left-handed superhelix. The Cse4-nucleosome has an extended conformation due to weaker DNA-histone interactions in the peripheral region of the nucleosome. Cse4 does not intrinsically prefer its native CEN DNA. Moreover, we found that $\mathrm{Scm} 3$ is not an integral component of Cse4-nucleosmes; rather, it functions as a Cse4-specific nucleosome assembly factor.

\section{Results}

Cse4-nucleosomes exhibit an elongated structure. To investigate the structural features of $S$. cerevisiae centromeric nucleosomes, we refolded recombinant yeast $\mathrm{H} 2 \mathrm{~A}, \mathrm{H} 2 \mathrm{~B}$ and $\mathrm{H} 4$ with either recombinant $\mathrm{H} 3$ or its centromere-specific equivalent $\mathrm{Cse} 4$. Histone octamers with full-length or N-terminal tail deletions $\left(\mathrm{Cse}_{\Delta 102}\right.$, $\mathrm{Cse} 4_{\Delta 127}$ ) were also prepared. $\mathrm{Cse}_{\Delta 127}$ corresponds to the structured region of $\mathrm{H} 3$, whereas $\mathrm{Cse}_{\Delta 102}$ has an $\mathrm{N}$-terminal tail that is similar in length to that of yeast H3 (Supplementary Fig. S1a). All histone octamers eluted at the expected volume from the gel-filtration column (Supplementary Fig. S1b) and contained equimolar amounts of the four histones (Supplementary Fig. S1d).

Nucleosomes were reconstituted onto a $147 \mathrm{bp}$ fragment derived from $\alpha$-satellite $\mathrm{DNA}^{27}$ by salt deposition, and analysed by native polyacrylamide gel electrophoresis (PAGE). Nucleosomes reconstituted from $S$. cerevisiae histones (Sc-Nuc) migrate similarly to nucleosomes made with Xenopus laevis histones (Xl-Nuc; Fig. 1a, lanes 3 and 6). In contrast, nucleosomes reconstituted with yeast $\mathrm{H} 2 \mathrm{~A}, \mathrm{H} 2 \mathrm{~B}, \mathrm{H} 4$ and $\mathrm{Cse} 4$ (Cse4-Nuc) migrate more slowly on native gels (Fig. 1a, lane 2). This property is not a function of the extended $\mathrm{N}$-terminal tail of Cse4, as partial or complete deletion of the Cse4 tail had little effect on gel migration (Fig. 1a, compare lanes 4 and 5 with lane 2). Similar gel mobility properties were also observed for Cse4-nucleosomes reconstituted onto $S$. cerevisiae centromeric DNA (147-bp CEN3) and a 147 bp fragment of the '601' nucleosome positioning sequence (601 DNA) ${ }^{28}$ (Fig. 1a, compare lanes 7 and 8 with 10 and 11; See Supplementary Table S1 for all DNA sequences). We analysed the protein content of the main, most intense band for each nucleosome sample excised from the gel shown in Figure 1a. The reconstituted nucleosomes contained equimolar amounts of all four histones (Cse4, H4, H2A and H2B; Fig. 1b). This was verified by size-exclusion chromatography coupled with multiangle light scattering (SEC-MALS). The molecular weight of all nucleosomes was fully consistent with a monodisperse complex of two copies of each histone and $147 \mathrm{bp}$ of DNA (Table 1 and Supplementary Fig. S2b).

We next wanted to examine whether the incorporation of Cse 4 causes a reversal in the nucleosomal DNA superhelix handedness in Cse4-nucleosomes, as proposed previously ${ }^{29}$. Both Cse4- and Scnucleosomes were assembled onto relaxed plasmid DNA at low salt using the yeast histone chaperone Nap1 (yNap1). Assemblies were analysed by agarose gel electrophoresis in the absence and presence of chloroquine (Fig. 1c). The data show that both Sc- and Cse4nucleosomes induce mainly negative supercoils. This was confirmed with a two-dimensional gel system first in the absence, then in the presence of chloroquine. This approach allows for the separation of mixtures of positively and negatively supercoiled DNA (Fig. 1f-i). 

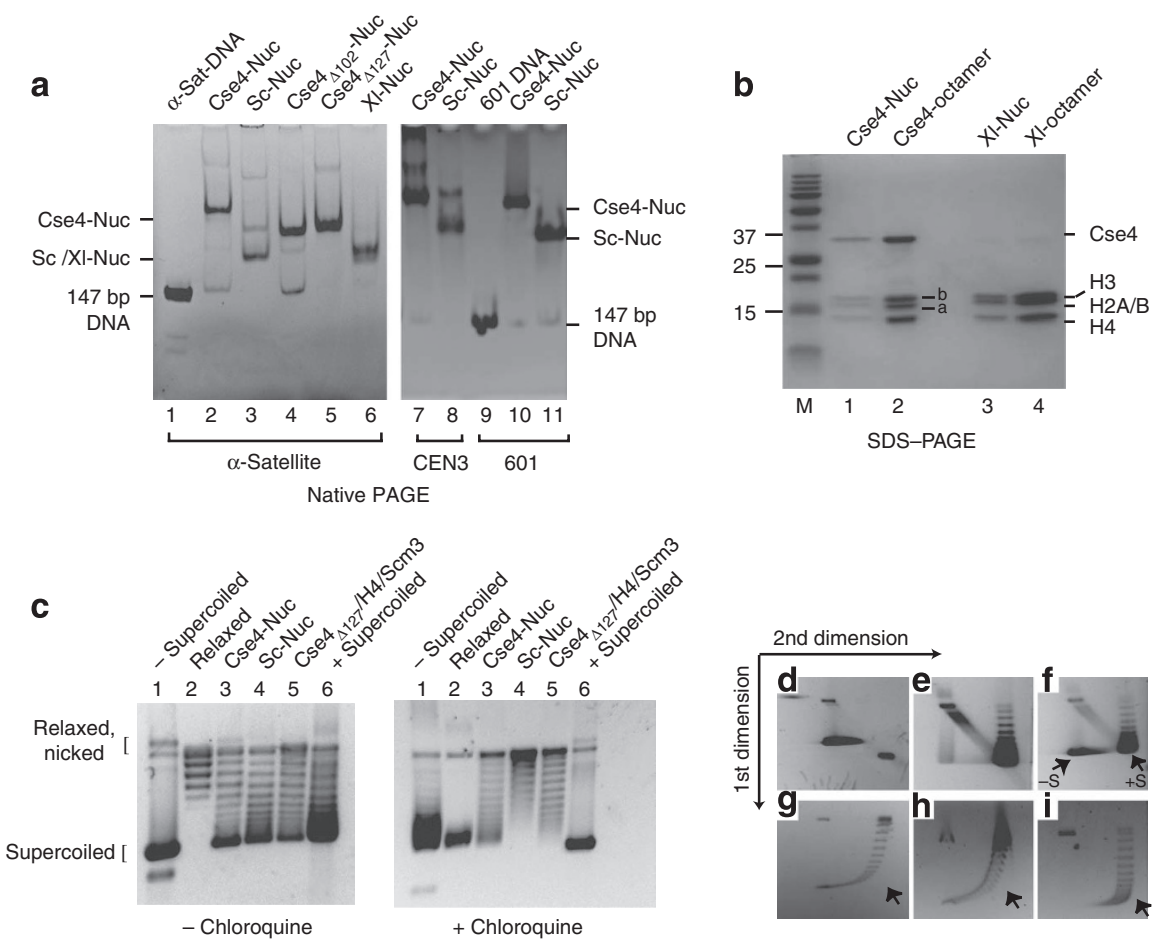

Figure 1 | In vitro assembled Cse4-containing nucleosomes exhibit retarded gel mobility and organize DNA in left-handed superhelix. (a) Cse4nucleosomes were reconstituted onto 147-bp DNA segments by salt dilution and analysed by $5 \%$ native PAGE followed by ethidium bromide staining. Lane 1: 147-bp $\alpha$-satellite DNA; lanes 2, 4 and 5: nucleosomes with full-length Cse4, Cse $4_{\Delta 102}$ and $C_{s e} 4_{\Delta 127}$, respectively, assembled on $\alpha$-sat DNA; lanes 3 and 6: S. cerevisiae (Sc) and Xenopus laevis (XI) nucleosomes on $\alpha$-sat DNA, respectively. Lanes 7 and 8: Cse4- and Sc-nucleosomes reconstituted on yeast centromeric DNA (CEN3); lane 9: 147-bp 601 DNA; lanes 10 and 11: Cse4- and XI-nucleosomes reconstituted on 147-bp 601 DNA. (b) Nucleosome bands were eluted from the gel shown in a, lanes 2 and 6, and analysed by SDS-PAGE and Coomassie Blue staining. Lanes 1 and 3: Cse4- and XInucleosome bands; 2 and 4: Cse4- and XI-octamers; M: protein molecular weight standards. Bands indicated as 'a': yeast H2A; 'b': yeast H2B. (c-i) Cse4nucleosomes induce negative supercoiling: (c) Nucleosomes were assembled on a circular pBR322 plasmid using yNap1 in the presence of Topoisomerase

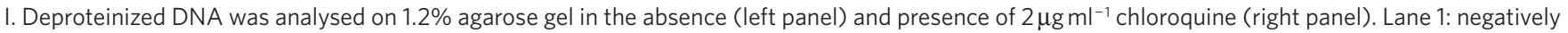
supercoiled plasmid; lane 2: relaxed plasmid; lanes 3 and 4: Cse4- and Sc-nucleosomes, respectively; lane 5: $\mathrm{Cse}_{\Delta 122} / \mathrm{H} 4 / \mathrm{Scm} 3$; lane 6: positively supercoiled pBR322 plasmid (purchased from Inspiralis). (d-i) Two-dimensional gel analysis of plasmid supercoiling. (d) and (e): negatively and positively supercoiled plasmid DNA, respectively, (f): samples in d, e combined; plasmid supercoiling induced by $\mathrm{Cse}$-nucleosomes $(\mathbf{g}), \mathrm{Cse}_{\Delta 127} / \mathrm{H} 4 / \mathrm{Scm} 3 / \mathrm{DNA}$ complex (h) and Sc-nucleosomes (i). $-\mathbf{S}$ and $+\mathrm{S}$ refer to negatively and positively supercoiled plasmid DNA. The arrow in $\mathbf{g}-\mathbf{i}$ indicates wherein the most supercoiled topoisomers run if they were positively supercoiled.

\section{Table 1 | SEC-MALS molecular weight estimation of canonical and Cse4-containing nucleosomes.}

\begin{tabular}{lcc} 
Nucleosome & $\begin{array}{c}\text { SEC-MALS molecular } \\
\text { mass (kDa) }\end{array}$ & $\begin{array}{c}\text { Calculated } \\
\text { molecular mass } \\
\text { (kDa) }\end{array}$ \\
\hline XI-Nuc & $200.0(0.6 \%)$ & 205 \\
Sc-Nuc & $202.3(1.0 \%)$ & 205 \\
Cse4-Nuc & $233.6(3.5 \%)$ & 223 \\
Cse4 $_{\Delta 102}$-Nuc & $208.0(0.6 \%)$ & 204 \\
\hline
\end{tabular}

Size-exclusion chromatography (SEC) in combination with multiangle light scattering (MALS) was used to analyse the monodispersity and molecular weight of the samples. Calculated molecular masses are given for a histone octamer with $147 \mathrm{bp}$ of DNA; values are derived from the average of three experiments. Note that the values given in Supplementary Fig. S2b are from single representative experiment.

Cse4- and Sc-nucleosomes exhibited identical behaviour (Fig. 1g,i), in that the vast majority of supercoiled plasmid fractions were negatively supercoiled.

To further investigate the structural differences resulting from the incorporation of Cse4, we used small-angle X-ray scattering (SAXS). We compared Xl-, Sc- and Cse4-nucleosomes assembled on 147-bp 601 DNA. To minimize effects caused by the differences in tail length between yeast H3 and Cse4 (Supplementary Fig. S1a), we used the truncated version of Cse $4\left(\mathrm{Cse}_{\Delta 102}\right)$. As shown above, partial deletion of the Cse 4 N-terminal tail does not significantly affect the slow gel migration signature of $\mathrm{Cse}_{\Delta 102}$ nucleosomes. All samples were shown to be monodisperse by native PAGE and SECMALS before data collection (Supplementary Fig. S2a,b).

A comparison of the scaled intensity curves of the three nucleosomes indicates clear differences between $\mathrm{Cse}_{\Delta 102}$-nucleosomes and canonical nucleosomes (Fig. 2a). The $\mathrm{P}(\mathrm{r})$ functions have the characteristic bell shape of globular complexes with well-defined maximum particle sizes for all three nucleosome preparations (Fig. $2 \mathrm{~b})$. The radius of gyration $\left(R_{\mathrm{g}}\right)$ and maximum dimensions $\left(D_{\max }\right)$ for Xl-nucleosomes were 41 and $120 \AA$, respectively (Fig. $2 \mathrm{~b}$ and Supplementary Table S2). The scattering curve calculated from the crystal structure ( $1 \mathrm{kx} 5$; ref. 30$)$ superposes well with the experimental scattering curve of Xl-nucleosomes (Fig. 2c). The ab initio calculated envelope for Xl-nucleosome exhibits excellent shape and size correspondence with the crystal structure (Supplementary Fig. S2c). Sc-nucleosomes are characterized by a slightly increased $R_{\mathrm{g}}(\sim 42.5 \AA)$ and a $D_{\max }$ of $\sim 135 \AA$, but the experimental scattering curve remains in close correspondence with the scattering curve calculated from the crystal structure (Fig. 2d); however, the envelope of Sc-nucleosome is somewhat extended (Supplementary Fig. S2d). The replacement of yeast $\mathrm{H} 3$ with $\mathrm{Cse} 4_{\Delta 102}$ resulted in an even more extended 

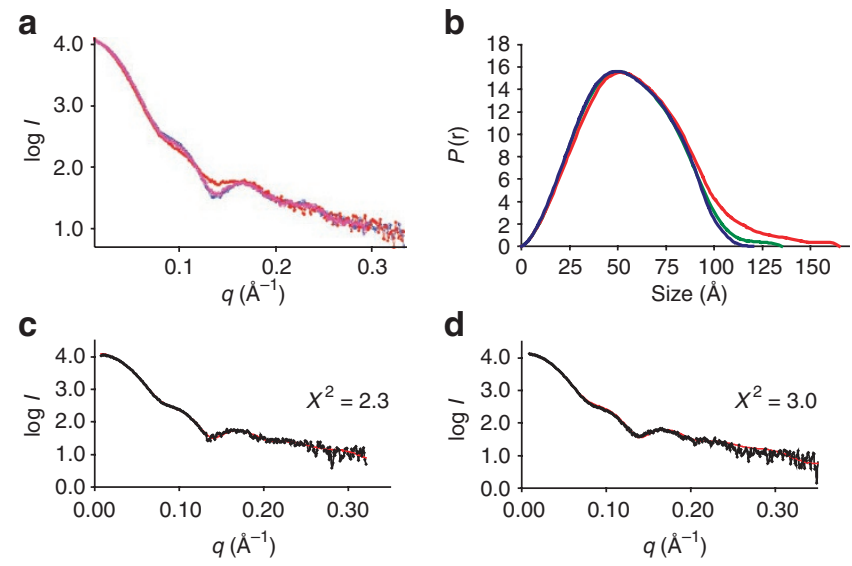

d
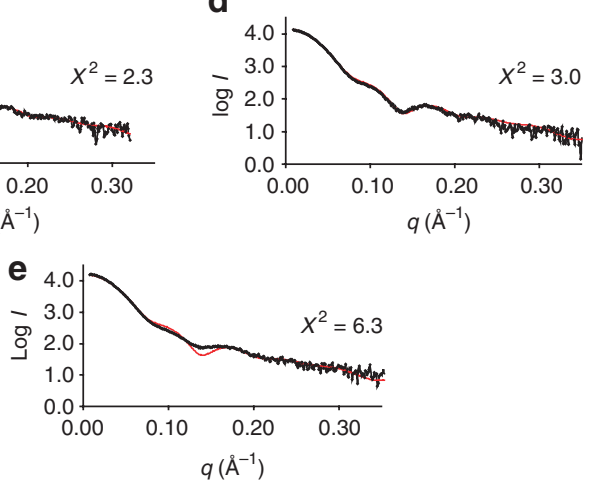

Figure 2 | Cse4-nucleosomes exhibit an extended morphology.

(a) Normalized SAXS curves of nucleosomes. $\mathrm{Cse}_{\triangle 102}$-nucleosomes (red) produce a clearly different scattering curve compared with Sc-nucleosomes (magenta) or Xl-nucleosomes (blue). (b) Distance distribution functions $(P(r))$ of the three nucleosomes shown in $\mathbf{a}$.

$\mathrm{XI}$-nucleosome is shown in blue, Sc-nucleosome in green and $\mathrm{Cse}_{\Delta 102^{-}}$ nucleosomes in red. Differences in the maximum dimensions of the three nucleosomes (see also Supplementary Table S2) are obvious. Ab initio envelopes, calculated from the intensity data, are shown in Supplementary Figure S2. (c-e) Comparison of experimental scattering profiles (black) with a scattering profile calculated using FoXS ${ }^{51}$ from the $\mathrm{XI}$-nucleosome crystal structure (1kx5; red). Experimental profiles from XI-, Sc- and $\mathrm{Cse}_{\Delta 102}$-nucleosomes are in c-e, respectively. The $\chi^{2}$ values of the fit between the two curves are shown.

structure $\left(R_{\mathrm{g}} \sim 46.5 \AA{ }^{\prime} ; D_{\max } \sim 165 \AA\right.$; Fig. $2 \mathrm{~b}$ and Supplementary Table $\mathrm{S} 2$ ), as well as in distinct differences in the intensity curves (Fig. 2e). The magnitude of the observed differences for nucleosomes with different conformations is consistent with those published earlier by us and others ${ }^{31,32}$. The calculated envelope for Cse $4_{\Delta 102}$-nucleosomes can be fit with a nucleosome, but the envelope displays pronounced protrusions (Supplementary Fig. S2e). This is consistent with the slow gel mobility of Cse4-nucleosomes. Together, our data demonstrate that the incorporation of Cse4 results in nucleosomes that organize DNA in a canonical negative supercoil around a histone octamer, and that these nucleosomes are somewhat extended.

The Cse4-nucleosome organizes only 115 bp of DNA. The observed characteristics of the Cse4-nucleosome could be because of less tightly bound DNA at the entry/exit regions. To test this, micrococcal nuclease (MNase) digestion of Cse4- and Sc-nucleosomes was conducted. Nucleosomes were reconstituted on either a linearized plasmid DNA using Nap1 or onto a 147-bp 601 DNA fragment by salt dilution. Cse4-nucleosomes offer less resistance towards MNase digestion than do Sc-nucleosomes, and protect only $\sim 110-120$ bp of DNA (Supplementary Fig. S3). This finding correlates with a recent report, wherein salt-reconstituted Cse4 nucleosomes were shown to protect $\sim 125 \mathrm{bp}^{33}$.

We used a single-molecule DNA-unzipping approach to provide a direct comparison of histone-DNA interactions in a Cse4nucleosome compared with an Sc-nucleosome. A DNA molecule containing either a single Cse4- or Sc-nucleosome assembled onto
601 DNA was attached to the surface of a microscope coverslip via one of its strands and to a microsphere held in an optical trap via the other strand (Supplementary Fig. S4). The magnitude of resistance towards unzipping strongly correlates with the strength of histoneDNA interactions, and thus a histone-DNA interaction map can be generated along the $\mathrm{DNA}^{34}$.

We first examined Sc-nucleosomes by unzipping DNA from either the forward or reverse direction along the DNA sequence (Fig. 3, left panel). Two distinct regions of interactions were detected; contacts between the $\mathrm{H} 2 \mathrm{~A} / \mathrm{H} 2 \mathrm{~B}$ dimers and the DNA (super-helical location (SHL) $\sim 3.5$ to \pm 6.5$)$; and contacts between the $(\mathrm{H} 3 / \mathrm{H} 4)_{2}$ tetramer and the DNA (SHL $\sim-2.5$ to +2.5 ). Within each region, interactions were discretely spaced with $\sim 5$ bp periodicity (Supplementary Figs S5 and 6). Signatures from recombinant yeast nucleosomes are indistinguishable from those obtained with nucleosomes from native $\mathrm{HeLa}$ histones $^{34}$. Unzipping through a DNA molecule assembled with a tetramer exhibited only a single region of interaction that substantially overlapped with the SHL -2.5 to 2.5 region where $(\mathrm{H} 3 / \mathrm{H} 4)_{2}$ tetramer contacts DNA in canonical nucleosomes (Supplementary Fig. S5).

The unzipping signature of Cse4-nucleosomes resembles that of Sc-nucleosomes (Fig. 3, right panel and Supplementary Fig. S6). However, the peak forces in SHL 3.5-6.5 regions in both sides of the nucleosomes were significantly lower than those of Sc-nucleosomes, suggesting that the DNA is more weakly bound to this region of the Cse 4 histone octamer. Unexpectedly, the histone-DNA interactions in SHL $\sim-2.5$ to 2.5 region (flanking the dyad by $\sim 30 \mathrm{bp}$ ), which involves the majority of direct contributions from $\mathrm{H} 3$ or Cse4, is at least as tight as in Sc-nucleosomes (Fig. 3). The force required for the unzipping fork to overcome the second region from the reverse direction is slightly higher for Cse4-nucleosomes, indicating a small asymmetric Cse4-induced increase in interaction strength at the tetramer. Taken together, this indicates that the overall structure of a centromeric nucleosome resembles that of a canonical nucleosome except for the more loosely bound outer $\sim 2 \times 2=40$ bp of DNA.

Cse4-octamers do not preferentially assemble on CEN3 DNA. S. cerevisiae centromeric DNA is highly AT-rich, making it a poor substrate for nucleosome formation ${ }^{35}$. Nevertheless, Cse4- and Scnucleosomes can be reconstituted on CEN3 DNA (Fig. 1a, lanes 7 and 8). We wanted to examine whether Cse4-octamers preferentially assemble on CEN3 DNA. Using salt deposition, we reconstituted a limiting amount of histone octamers on a cy3-labelled 207-bp 601 or atto647-labelled 207-bp CEN3 DNA fragment in the presence of an excess of unlabelled 147-bp 5S DNA (see experimental scheme in Fig. 4a). The fraction of DNA incorporated into nucleosomes (using data from Fig. 4b-d with histone-DNA ratio of 0.32:1 for Cse4octamer, and 0.28:1 for Sc-octamer) was quantified (Fig. 4e). Both Cse4- and Sc-octamers preferentially assemble on 601 DNA compared with 5S DNA, confirming the strong positioning propensities of this sequence ${ }^{28}$. Both octamers assembled much less efficiently on CEN3 DNA and 5S DNA, demonstrating that the Cse4-octamer does not have an intrinsic preference for its native CEN DNA.

To address the relative stability of Cse4- and Sc- nucleosomes reconstituted on various DNA fragments, we assayed their integrity after storage on ice for at least $24 \mathrm{~h}$ (Supplementary Fig. S7). Cse4and Sc-nucleosomes are equally stable when reconstituted on 601 DNA, and display no signs of dissociation after 1 week of storage on ice (Supplementary Fig. S7a). Similarly, Cse4-nucleosome reconstituted onto a generic 147-bp DNA fragment derived from pBR322 remains intact on storage (Supplementary Fig. S7d). In contrast, a Cse4-nucleosome reconstituted on its cognate CEN-DNA dissociates almost completely after $24 \mathrm{~h}$ of storage, whereas a Sc-nucleosome on the same DNA or a Cse4-nucleosome on 5S DNA remains largely intact (Supplementary Fig. S7b,c). Together, this data suggests that Cse 4 and CEN DNA might have co-evolved to be particularly unstable in the absence of other centromere-specific factors. 

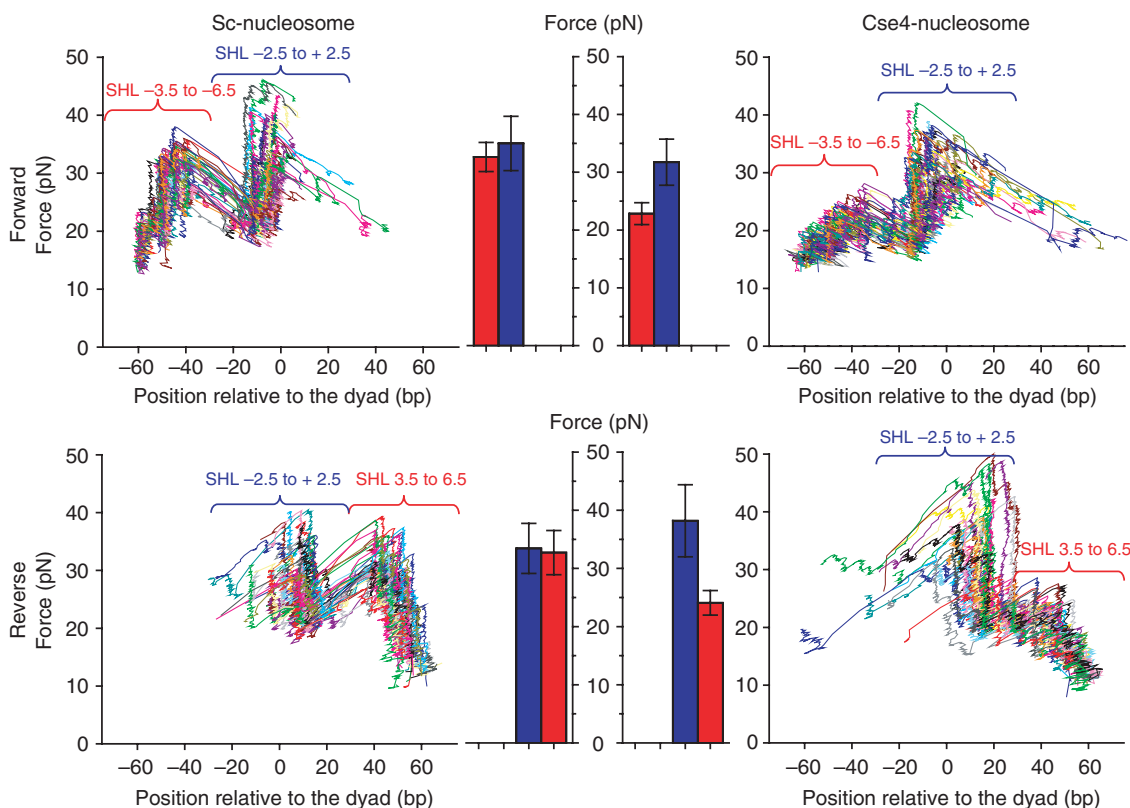

Figure 3 | Single-molecule analysis shows that Cse4-nucleosomes have weakened histone-DNA interactions in the peripheral regions. Nucleosome disruption signatures under a constant loading rate $\left(8 \mathrm{pN} \mathrm{s}^{-1}\right)$. Representative traces are shown for canonical Sc-nucleosomes (left panel) and Cse4nucleosomes (right panel), where each colour represents data obtained from a single-nucleosomal DNA molecule. Interaction regions are defined according to the crystal structure ${ }^{27}$ as super-helical location $(\mathrm{SHL})-2.5$ to $+2.5(\mathrm{H} 3 / \mathrm{H} 4$ tetramer interaction region) and $\mathrm{SHL} \pm 3.5$ to $\pm 6.5(\mathrm{H} 2 \mathrm{~A} / \mathrm{H} 2 \mathrm{~B}$ dimer interaction region). Within the SHL -2.5 to +2.5 region the maximum disruption forces observed are similar between Sc- and Cse4-nucleosomes. Cse4-nucleosomes have significantly weaker interactions throughout the peripheral regions ( $\mathrm{SHL} \pm 3.5$ to \pm 6.5 ). A quantitative comparison of the peak forces measured within each interaction region is shown (centre panel). SHL \pm 3.5 to \pm 6.5 is shown in red bar and $\mathrm{SHL}-2.5$ to +2.5 shown in blue. Error bars represent s.d. See Supplementary Figures S4-6 for additional data.

$\mathrm{Scm} 3$ is not a component of the Cse4-nucleosome. It has been proposed that the putative centromeric histone chaperone $\mathrm{Scm} 3$ replaces histones $\mathrm{H} 2 \mathrm{~A} / \mathrm{H} 2 \mathrm{~B}$ dimers in a Cse4-containing nucleosome-like particle. Scm 3 can be refolded with $\mathrm{Cse} 4$ and $\mathrm{H} 4$ into a well-defined and monodisperse complex (ref. 11 and Supplementary Fig. S1c,d). We tested whether this $\mathrm{Cse} 4_{\Delta 127} / \mathrm{H} 4 / \mathrm{Scm} 3$ complex can be reconstituted into $\mathrm{Scm} 3$-containing nucleosome-like particles. The refolded $\mathrm{Cse} 4_{\Delta 127} / \mathrm{H} 4 / \mathrm{Scm} 3$ complex was reconstituted on 207-bp 601 DNA using salt dilution. The resulting species exhibited variable electrophoretic mobility (Fig. 5a). The major (and in case of lane 5 , minor) bands were eluted from the gel and analysed by SDS-PAGE (Fig. 5b). Only $\left(\mathrm{Cse}_{\Delta 127} / \mathrm{H} 4\right)_{2}$, but not $\mathrm{Scm} 3$, is bound to DNA in any of these complexes (Fig. 5b, lanes 6 and 7). When the same reaction was repeated in the presence of $\mathrm{H} 2 \mathrm{~A} / \mathrm{H} 2 \mathrm{~B}$ dimer, an octameric nucleosome complex consisting of Cse4, H4, H2A and $\mathrm{H} 2 \mathrm{~B}$ was formed (data not shown).

To exclude the possibility that $\mathrm{Scm} 3$ dissociated from the complex during gel electrophoresis, we examined the products of saltmediated $\mathrm{Cse} 4_{\Delta 127} / \mathrm{H} 4 / \mathrm{Scm} 3 / \mathrm{DNA}$ reconstitution by sucrose gradient sedimentation analysis. The slow migrating bands (indicated by ' ${ }^{\prime}$ ) were not stable and dissociated during sedimentation (Fig. $5 \mathrm{c}$ upper panel); these bands are likely additional $\mathrm{Cse}_{\Delta 127} / \mathrm{H} 4$ in loose association with the Cse $4_{\Delta 127} / \mathrm{H} 4 / \mathrm{DNA}$ complex (tetrasome). $\left(\mathrm{Cse} 4_{\Delta 127} / \mathrm{H} 4\right)_{2}$ tetrasome and $\mathrm{Scm} 3$ elute separately in the sucrose gradient (Fig. 5c lower panel), confirming that $\mathrm{Scm} 3$ is not stably associated with the $\mathrm{Cse}_{\Delta 127} / \mathrm{H} 4 / \mathrm{DNA}$ complex. Control Cse $4_{\Delta 127^{-}}$ nucleosomes reconstituted on the same DNA contained all four histones $\left(\mathrm{Cse}_{\Delta 127}, \mathrm{H} 4, \mathrm{H} 2 \mathrm{~A}\right.$ and H2B; Supplementary Fig. S8), demonstrating that the $\mathrm{Cse} 4_{\Delta 127}$-nucleosomes were stable during sucrose gradient sedimentation. We also investigated the superhelical organization of particles reconstituted from refolded Cse $4_{\Delta 127} / \mathrm{H} 4 /$ $\mathrm{Scm} 3$ using a plasmid supercoiling assay. This complex introduced predominantly negative supercoils into plasmid DNA (Fig. 1c, lane
5 in left and right panels, and Fig. 1h). This shows that the canonical handedness of the DNA supercoil is maintained even in the absence of $\mathrm{H} 2 \mathrm{~A} / \mathrm{H} 2 \mathrm{~B}$ dimer and in presence of $\mathrm{Scm} 3$.

Scm3 functions as a Cse4-specific nucleosome assembly factor. $\mathrm{Scm} 3$ is required for $\mathrm{CenH} 3$ deposition on the centromere in both S. cerevisiae and Schizosaccharomyces pombe $e^{11,12,21,22}$. We investigated whether $\mathrm{Scm} 3$ mediates Cse4-nucleosome assembly in vitro. We also wanted to exclude the possibility that under these physiological (as opposed to high-ionic strength) conditions $\mathrm{Scm} 3$ remains an integral part of the yeast centromeric nucleosome ${ }^{10}$ ), perhaps altering the handedness of the resulting nucleosomes, as observed in $v i v o^{13}$. We expressed a truncated version of $\mathrm{Scm} 3$, encompassing the conserved Cse4-binding region of $\mathrm{Scm} 3$ (amino acids 63-189; $\left.\mathrm{Scm} 3_{63-189}\right)^{11,36}$ and full-length $\mathrm{Scm} 3$ in Escherichia coli (Supplementary Fig. S1e). In our standard chaperone-mediated chromatin assembly assay, we used $\mathrm{Scm} 3$ or $\mathrm{Scm} 3_{63-189}$ (in place of yNAP1) on a plasmid containing four copies of 190-bp CEN3 and four copies of CEN, followed by an analysis of supercoiling (Fig. 6a).

$\mathrm{Scm} 3$ clearly assembles Cse4-nucleosomes, and the conserved region $\left(\mathrm{Scm}_{63-189}\right)$ is sufficient for this activity (Fig. 6b left panel, compare lanes 4 and 5). $\mathrm{Scm}_{63-189}$ is specific for Cse4, as it showed only poor assembly of Sc-nucleosomes (Fig. 6c left panel)). As observed for Cse4-nucleosomes assembled by yNap1 (Fig. 1c), Cse4-nucleosomes reconstituted with $\mathrm{Scm} 3$ and $\mathrm{Scm}_{63-189}$ induced negative DNA supercoils into plasmids containing CEN sequences (Fig. 6b,c, right panels). Together, this data demonstrate that $\mathrm{Scm} 3$ has Cse4-specific histone chaperone activity that assembles nucleosomes with a left-handed DNA supercoil.

As shown above (Fig. 1a,b and Table 1), Cse4-nucleosomes reconstituted by a salt dilution approach in the absence of $\mathrm{Scm} 3$ are octameric in composition. We wanted to compare the composition of Cse4-nucleosomes reconstituted with yNap1 and $\mathrm{Scm} 3$ under 


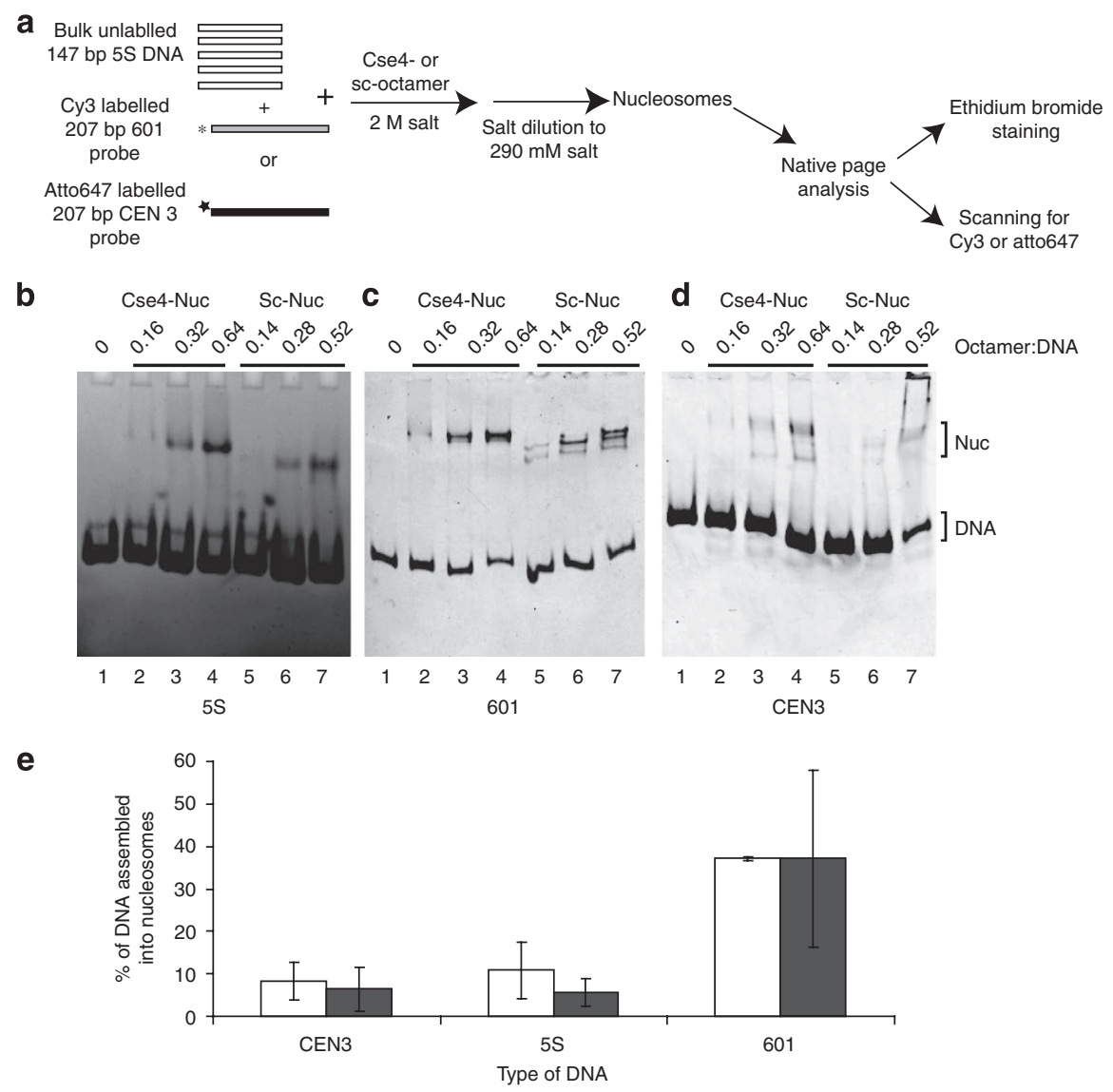

Figure 4 | Cse4-nucleosomes do not preferentially assemble on CEN3 DNA. (a) Experimental scheme of Cse4- and Sc-nucleosomes reconstitution on 5S, 601 and CEN3 DNA. Nucleosomes were reconstituted under limiting octamer concentration by salt dilution on either a 207-bp atto647-labelled CEN3 or cy3-labelled 601 DNA probe in the presence of an excess of 147-bp 5S DNA and analysed by 5\% native PAGE. (b) The reconstitution on total DNA $(\approx 5 S$ DNA) was visualized by ethidium bromide staining. Nucleosomes assembled on 601 DNA and CEN3 DNA were detected by scanning for cy3 601 (c) and atto647 (d), respectively. The ratio of octamer to DNA and the types of histone octamer (Cse4, Sc) used are indicated above the lanes. (e) Quantification of nucleosome assembly. The fraction of DNA incorporated into nucleosomes in $\mathbf{b}$-d was calculated by dividing the signal of the shifted band(s) by the sum of free DNA and shifted band signal, and multiplying by 100 . We excluded the material in the wells, but added all nucleosome bands. The graph of nucleosomes reconstituted using octamer-DNA ratio of 0.32:1 (Cse4-Nuc, shown in white) and 0.28:1 (Sc-nuc, shown in black) is shown. The mean and standard deviation values from 4 to 5 different experiments were used to plot the graph.

physiological ionic strength onto different DNA sequences $(601,5 \mathrm{~S}$, CEN3). Cse4-octamers were assembled onto linear 561-bp DNA (containing three 147-bp 601 segments connected by 60-bp linker DNA) in the presence of yNap1, $\mathrm{Scm} 3_{63-189}$ or Scm3. The assembled nucleosomes (likely a mixture between mono-, di- and trinucleosomes) were fractionated by sucrose gradient sedimentation and the fractions were analysed by native PAGE and SDS-PAGE (Fig. 6d,e and Supplementary Fig. S9). SDS-PAGE analysis of individual fractions demonstrates that Cse4-nucleosomes assembled on 601 DNA by yNap1 (Supplementary Fig. S9a), Scm3 (Supplementary Fig. S9b) or $\mathrm{Scm}_{63-189}$ (Fig. 6d) are composed of Cse4, H2A, H2B and H4. Neither yNap1, nor Scm3 or Scm3 ${ }_{63-189}$ co-fractionate with the nucleosome; both chaperones elute at significantly lower sucrose density and clearly separate from the nucleosomes. The yNap1 fractions that partially overlap with the nucleosome (Supplementary Fig. S9a, right panel, lane 13 and 16) are likely caused by the previously described oligomerization of yNap1 dimers at physiological ionic strength ${ }^{37}$. Cse4-nucleosomes were also assembled on 207-bp CEN3 and 207-bp 5S DNA fragments using $\mathrm{Scm}_{63-189}$ as the assembly factor and were shown to be octameric (Supplementary Fig. S9c,d). Together, our results suggest that under the conditions used, $\mathrm{Scm} 3$ is not integrated into Cse4-nucleosomes on any of the DNA sequences tested, including CEN DNA. Thus, we conclude that $\mathrm{Scm} 3$ is not a stable component of Cse4-nucleosomes but functions as Cse4-nucleosome-specific assembly factor.

\section{Discussion}

Here we have shown that Cse4-nucleosomes resemble canonical nucleosomes in their octameric histone composition and DNA organization into a left-handed DNA supercoil, as shown recently by analytical ultra centrifuge and atomic force microscope ${ }^{33}$. The intrinsic properties of Cse4-containing histone complexes are sufficient to determine these features, as salt- and chaperone-mediated assembly pathways on different DNA sequences (including CEN DNA) have the same outcome. The unique sequence features of CEN DNA do not confer preference for Cse4. Under no condition do we observe a nucleosome-like particle consisting of Cse4, $\mathrm{H} 4$ and $\mathrm{Scm} 3$; rather our results suggest a role for $\mathrm{Scm} 3$ as a Cse4-specific assembly factor. Despite the overall similarities, we observe significant structural deviations in Cse4-nucleosomes. They exhibit an open conformation that is at least partly due to weaker interactions in the DNA regions organized by the $\mathrm{H} 2 \mathrm{~A} / \mathrm{H} 2 \mathrm{~B}$ dimers, and by the $\mathrm{N}$-terminal $\alpha$-helix and the base of the N-terminal tail of Cse 4 .

DNA sequences with poly (dA:T) tracts (as in CEN DNA) are predicted to disfavour the formation of stable nucleosomes ${ }^{35}$. We demonstrate that the Cse4-octamer does not exhibit a preference 
a
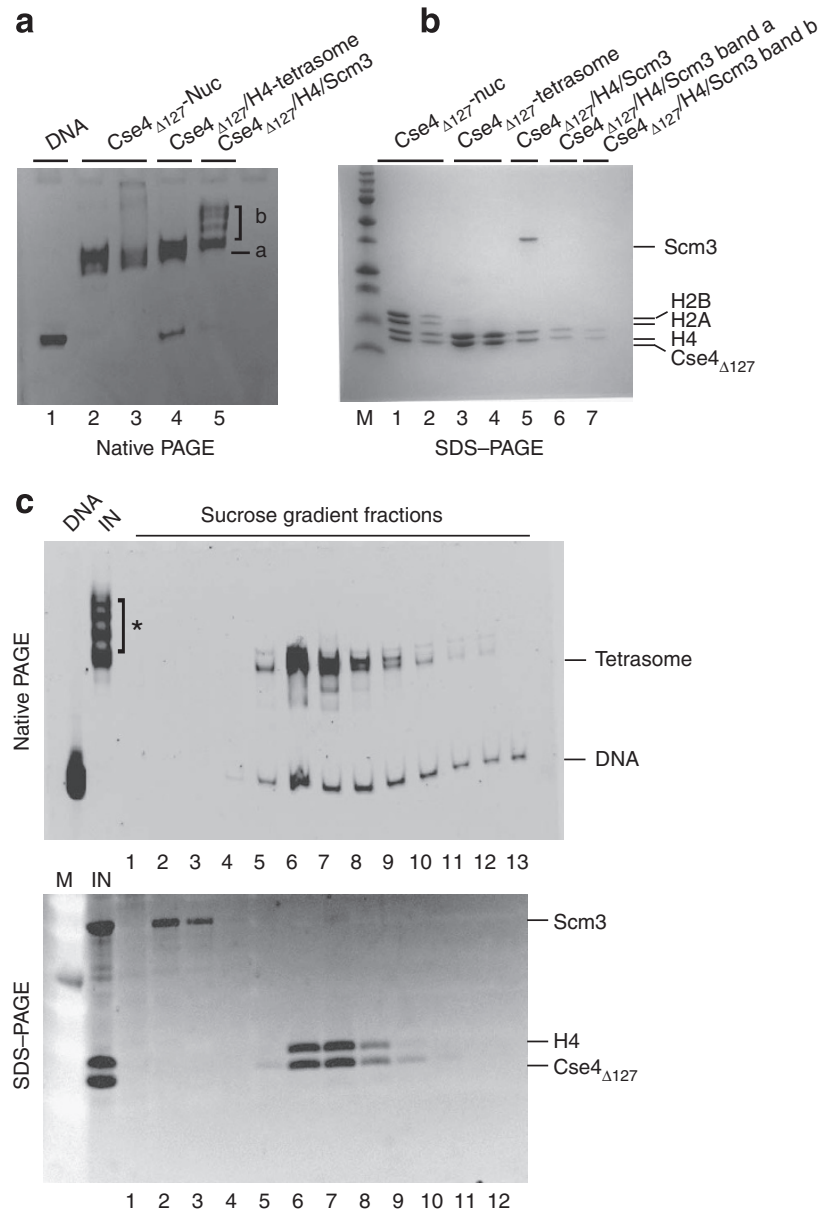

Figure 5 | Scm3 is not stably incorporated into nucleosome-like particles. (a) Two different concentrations of $\mathrm{Cse}_{\Delta 127}$-octamer (lanes 2 and 3), $\left(\mathrm{Cse}_{\Delta 127} / \mathrm{H} 4\right)_{2}$ tetramer (lane 4) and $\mathrm{Cse}_{\Delta 127} / \mathrm{H} 4 / \mathrm{Scm} 3$ (lane 5) were reconstituted on 601 DNA (207 bp) by salt dilution and analysed by gelshift on native 5\% PAGE. In lane 5, 'a' and 'b' refer to the lower discrete band and upper multiple bands, respectively. (b) Individual bands from gel shown in a were excised and analysed by $16 \%$ SDS-PAGE. Lanes 1: control $\mathrm{Cse}_{\Delta 127}$-nucleosome; 2: $\mathrm{Cse}_{\Delta 127}$-nucleosome band (from lane 3 in a); 3: control $\left(\mathrm{Cse}_{\Delta 127} / \mathrm{H} 4\right)_{2}$ tetrasome; 4 : $\left(\mathrm{Cse}_{\Delta 127} / \mathrm{H} 4\right)_{2}$ tetrasome band (lane 4 in a); 5 : control $\mathrm{Cse}_{\Delta 127} / \mathrm{H} 4 / \mathrm{Scm} 3$ complex; 6 and 7 correspond to the bands labelled as ' $a$ ' and ' $b$ ', respectively from lane 5 in $\mathbf{a}$. (c) Sucrose density gradient fractionation of $\mathrm{Cse}_{\Delta 127} / \mathrm{H} 4 / \mathrm{Scm} 3$ reconstituted on 601,207 bp DNA. Samples were fractionated in a 5-25\% sucrose density gradient. Upper panel: the fractions were analysed on $5 \%$ native PAGE and stained with ethidium bromide. Lanes: DNA: 207-bp 601; IN, input sample; 1-13: fractions from low- to high-density sucrose. Lower panel: samples 1-12 shown in the upper panel were analysed by SDS-PAGE stained with SYPRO Ruby (Invitrogen).

for CEN DNA, confirming interpretations from single-molecule

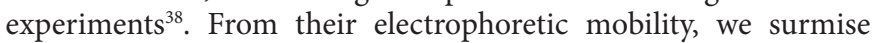
that Cse4-nucleosomes on CEN DNA also exist in the more open state observed for Cse4-nucleosomes on 601 and $\alpha$-sat DNA. Cse4nucleosomes on CEN3 DNA are particularly unstable on storage, and this may partly explain previous reports that Cse4-nucleosomes cannot be assembled on CEN DNA in vitro ${ }^{8}$, as well as the observed depletion of $\mathrm{H} 2 \mathrm{~A} / \mathrm{H} 2 \mathrm{~B}$, perhaps during preparation, from centromeric nucleosomes ${ }^{11}$. Thus, if there is a role for CEN DNA in modulating nucleosome structure at the yeast centromere, it is unlikely that it directly attracts $(\mathrm{Cse} 4 / \mathrm{H} 4)_{2}$, or that it forms particularly stable nucleosomes. Rather, the low propensity to form nucleosomes on CEN DNA suggests that centromeric sequences might have evolved to exclude any nucleosome formation to prevent incorporation of the abundant major-type H3. This allows for the targeted assembly of Cse4-nucleosomes only in the presence of centromeric proteins that are specific for Cse4 and CEN DNA. For example, the CBF3 complex interacts with Cbf1 and such interactions may be important to stabilize the observed 'open' centromeric nucleosomes. Thus, it seems that Cse4 has evolved to form a particularly unstable nucleosome on CEN DNA that is viable only in the presence of other key factors, ensuring proper centromere architecture. On the other hand, the more open architecture of Cse4-nucleosomes might generate an environment that is required for concurrent binding of centromere-associated proteins. Both CDE III and CDE I (the binding sites of CBF3 and Cbf1, respectively) are part of the 125-bp nucleosomal centromeric DNA. Assuming that Cse4-nucleosomes are also octameric in $v i v o^{8}$, partial dissociation of DNA ends would be required to allow binding of both CBF3 and Cbf1, as well as of other centromeric proteins ( $\mathrm{Scm} 3$ and Mif2). This may serve as the foundation of kinetochore assembly.

Our results favour a role for S. cerevisiae $\mathrm{Scm} 3$ as a Cse4/H4specific assembly factor but not as a component of the Cse4-nucleosome. As the effects of $\mathrm{scm} 3$ deletion can be rescued by overexpression of Cse4 (ref. 8), it is possible that Nap1 (which assembles Cse4 and $\mathrm{H} 3$-containing histone octamers equally well) or another chaperone replaces $\mathrm{Scm} 3$ in its Cse4-nucleosome assembly function. The structural basis for $\mathrm{Scm} 3$ specificity for $(\mathrm{Cse} 4 / \mathrm{H} 4)_{2}$ tetramer or in Cse4-nucleosome assembly needs further investigation. It has been suggested that $\mathrm{Scm} 3$ might displace $\mathrm{H} 2 \mathrm{~A} / \mathrm{H} 2 \mathrm{~B}$ dimers from a Cse4-nucleosome to form ( $\mathrm{Scm} 3 / \mathrm{Cse} 4 / \mathrm{H} 4)$-containing 'hexasomes ${ }^{311,38}$. We have shown that $\mathrm{Scm} 3$ does not remain associated with DNA when refolded Scm3/Cse4/H4 complex is reconstituted onto DNA using salt dialysis, and that addition of $\mathrm{H} 2 \mathrm{~A} / \mathrm{H} 2 \mathrm{~B}$ dimers to this reaction results in the formation of octameric nucleosomes. Octameric nucleosomes were also obtained when $\mathrm{Scm} 3$ was used as histone chaperone to assemble Cse4-nucleosomes under physiological salt conditions.

Together, our data favour a model in which $\mathrm{Scm} 3$ interacts with $\mathrm{CBF} 3$ and other centromeric proteins to facilitate the deposition of Cse $4 / \mathrm{H} 4$ on CEN DNA, resulting in the formation of an octameric Cse4-nucleosome. Once assembled, the intrinsic features of the Cse4-nucleosome on CEN DNA likely ensure that Cse4-nucleosomes (and not H3-nucleosomes) are only stable in the presence of other centromere-specific factors, ensuring proper centromere function.

\section{Methods}

DNA preparation. The various DNA sequences used for nucleosome reconstitution are listed in Supplementary Table S1. The DNA fragments were prepared by cutting the appropriate plasmids with the appropriate restriction enzymes or by PCR. $\alpha$-Satellite DNA (147bp) was prepared as previously reported ${ }^{39}$.

Protein purification and refolding. A pKS387-Cse 4 pET expression plasmid with a mutation in the NdeI site was a kind gift from the Harrison Laboratory. N-terminally truncated Cse 4 constructs (Cse4 103-229 and Cse4 128-229) were PCR amplified from pKS387-Cse4, and inserted into NdeI/BamHI sites of pKS387Cse4 plasmid replacing the full-length Cse4-coding sequence. Cse4 103-229 $\left(\mathrm{Cse} 4_{\Delta 102}\right)$ lacks the N-terminal 102 amino acids; the remaining $\mathrm{N}$-terminal tail is 9 amino acids shorter than that of canonical H3, whereas Cse4 128-229 $\left(\mathrm{Cse}_{\Delta 127}\right)$ is the equivalent of tailless canonical H3.

The Scm3-coding sequence was PCR amplified from yeast genomic DNA, and inserted into BspHI/EcoRI sites of pHAT2 plasmid vector DNA resulting in the addition of $\mathrm{His}_{6}$ to the $\mathrm{N}$-terminus. Similarly, the coding region for $\mathrm{Scm} 3_{63-189}$ was cloned into a pHAT4 plasmid vector and the protein was expressed with an $\mathrm{N}$-terminal $\mathrm{His}_{6}$-tag with a TEV cleavage site for removal of the tag.

Recombinant Cse4 and other yeast histone proteins were expressed in E. coli and purified as described previously ${ }^{40}$. Full-length $\mathrm{Scm} 3$ was purified from inclu-

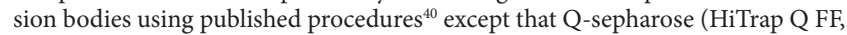
GE Healthcare) was used in the last step. Refolding of major-type and Cse4-containing histone octamers and tetramers, and of Cse $4 / \mathrm{H} 4 / \mathrm{Scm} 3$ complexes was done as described ${ }^{40}$. 
a
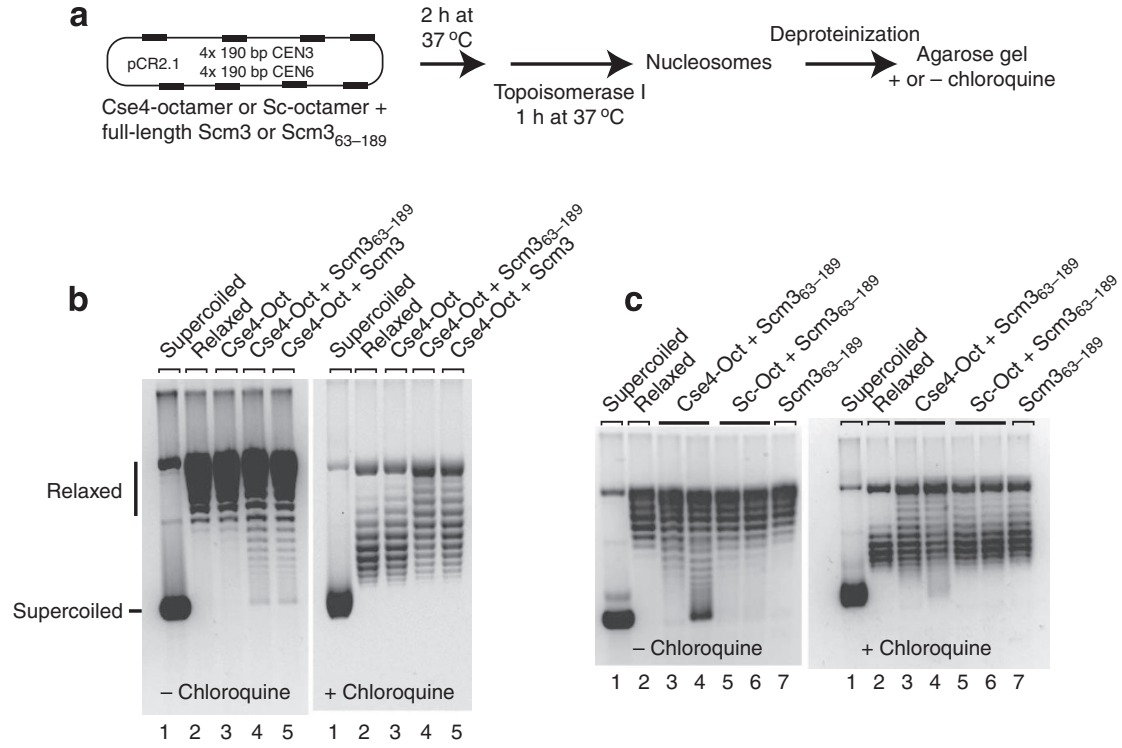

d
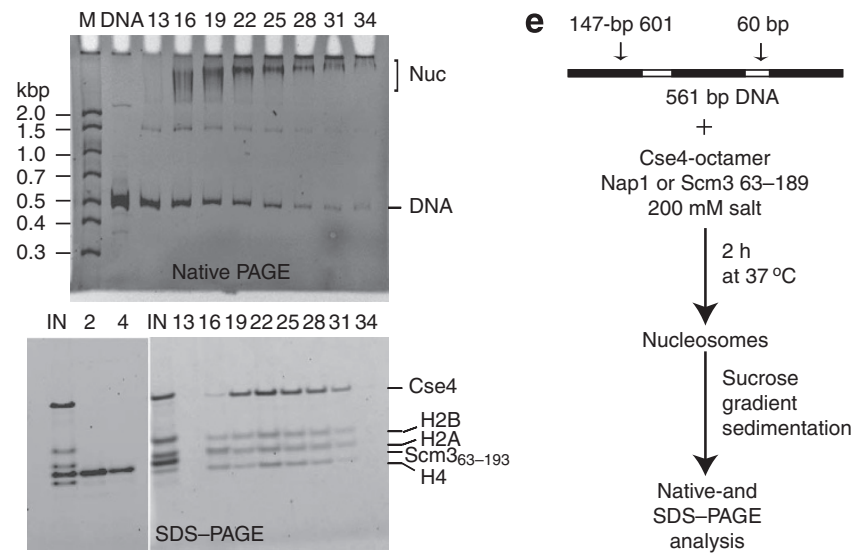

Figure 6 | Scm3 is a Cse4-specific chaperone that assembles nucleosomes with a negative supercoil. (a) As illustrated in the schematic, Cse4- or Sc-nucleosomes were assembled on relaxed circular plasmid DNA using full-length $\mathrm{Scm} 3$ or truncated $\mathrm{Scm}_{63-189}$ as a histone chaperone; nucleosome assembly was analysed by plasmid supercoiling assay. (b, c) Deproteinized DNA samples were analysed on $1 \%$ agarose gel in the absence and presence of chloroquine (as indicated), and visualized by SYBR Gold staining. (b) Full-length $\mathrm{Scm} 3$ and $\mathrm{Scm} 3_{63-189}$ assemble nucleosomes equally well. Lanes 1 and 2: negatively supercoiled or relaxed pCR2.1-(CEN3 + CEN6) 4 ; lane 3: Cse4-octamer (Oct) without histone chaperone; lanes 4 and 5: Cse4-octamer with 25 pmol Scm3 $_{63-189}$ and full-length Scm3, respectively. (c) Scm3 is specific for the Cse4-octamer: Cse4- or Sc-nucleosomes were assembled and analysed as described in a. Lanes 1 and 2: negatively supercoiled and relaxed plasmid, respectively; lanes 3 and 4: Cse4-nucleosome assembled in the presence of 25 and $50 \mathrm{pmol}$ of $\mathrm{Scm}_{63-189}$, respectively; lanes 5 and 6: Sc-nucleosomes assembled in the presence of 25 and 50 pmol Scm3 ${ }_{63-189,}$ respectively; lane 7: 50 pmol Scm3 63-189, $_{1}$ no histone octamer. (d) Cse4-nucleosomes assembled by Scm3 contain all four histones but not Scm3: As diagramed in e, Cse4nucleosomes were reconstituted on a 561-bp DNA containing three 147-bp 601 nucleosome positioning sequences using $\mathrm{Scm}_{63-189}$ as the chromatin assembly factor. The assembled chromatin was purified by sucrose gradient sedimentation. Incremental fractions 13-34 (top to bottom of the sucrose gradient) were analysed by $4 \%$ native PAGE and visualized by ethidium bromide (upper panel). Input samples and fractions 2,4 , and incremental fractions13-34 were analysed by SDS-PAGE stained with SYPRO Ruby (Invitrogen; lower panel). Control experiments with Nap1 instead of Scm3 ${ }_{63-189}$ as the assembly factor and assembly by full-length Scm3, and assembly on 207-bp CEN3 and 5S DNA by Scm3 ${ }_{63-189}$ are shown in Supplementary Figure S9. (e) Experimental scheme of nucleosome reconstitution for data shown in $\mathbf{d}$.

The full-length $\mathrm{Scm} 3$ was refolded in absence of $\mathrm{Cse} 4$ and $\mathrm{H} 4$ into a soluble state. Briefly, purified $\mathrm{Scm} 3$ was dissolved in buffer composed of $7 \mathrm{M}$ guanidine$\mathrm{HCl}, 20 \mathrm{mM}$ Tris-HCl pH 8, $1 \mathrm{mM}$ EDTA, $5 \mathrm{mM}$ dithiothreitol (DTT), 20\%glycerol and $0.01 \% \mathrm{NP} 40$. The sample was then dialysed against buffer consisting of $20 \mathrm{mM}$ Tris- $\mathrm{HCl} \mathrm{pH} 8,0.24 \mathrm{mM}$ tris (2-carboxyethyl) phosphine, $300 \mathrm{mM} \mathrm{NaCl}, 0.2 \mathrm{mM}$ PMSF, $0.3 \mathrm{mM}$ benzamidine, $20 \%$ glycerol and $0.01 \%$ NP40.

Truncated $\mathrm{His}_{6}$-tagged $\mathrm{Scm}_{63-189}$ was purified by Ni-NTA under native conditions. The $\mathrm{His}_{6}$-tag was removed with TEV protease, and the protein was further purified by gel-filtration using Superdex 75 in buffer containing $20 \mathrm{mM}$ Tris-HCl pH 8, $300 \mathrm{mM} \mathrm{NaCl}, 1 \mathrm{mM}$ pepstatin, $0.2 \mathrm{mM}$ PMSF, $1 \mathrm{mM}$ benzamidin and $0.01 \%$ NP40. The salt concentration was adjusted to $50 \mathrm{mM}$ and was further purified over a HiTrap Q FF column (GE Healthcare).

Nucleosome reconstitution by salt dilution. Cse4-, Xenopus laevis (Xl-) and S. cerevisiae (Sc-) nucleosomes were reconstituted on 147- and 207-bp DNA (5S,
601, CEN3, pBR322 DNA; sequences given in the Supplementary Table S1) using standard salt dilution or dialysis ${ }^{40}$, and analysed by $5 \%$ native PAGE and ethidium bromide staining. The histone composition of Cse4-nucleosomes was determined by electro-eluting the nucleosome bands from a native PAGE, and examining their composition by SDS-PAGE. The stability of Cse4-nucleosome was determining by analysing nucleosome sample stored at $4^{\circ} \mathrm{C}$ for $24 \mathrm{~h}$ or longer.

Competitive nucleosome reconstitution. Cse4-octamer (1.2-4.8 $\mu \mathrm{g})$ or Sc-octamer $(1.05-4.2 \mu \mathrm{g})$ was reconstituted by salt dilution onto $7.5 \mu \mathrm{g} 146-\mathrm{bp} 5 \mathrm{~S}$ DNA in the presence of limiting amounts ( 200 ng) of Cy3-labelled 207-bp 601 DNA or Atto647-labelled CEN3 DNA. The samples were separated on a 5\% native PAGE and visualized by ethidium bromide staining or scanned for fluorescence at the appropriate wavelength. The propensity of nucleosome formation on the different DNA sequences was determined by quantifying the percentage of 5S DNA or labelled DNA probe incorporated into nucleosomes. 
Histone chaperone-mediated nucleosome reconstitution. Nucleosomes were reconstituted on relaxed circular plasmid or linear DNA using a histone chaperone $e^{41}$ with some modifications. In a typical $30 \mu$ reaction, histone octamer (12.5 pmol) was preincubated for $10 \mathrm{~min}$ at $37^{\circ} \mathrm{C}$ with $25-50$ pmol yeast Napl (yNap1) or full-length $\mathrm{Scm} 3$ or $\mathrm{Scm}_{63-189}$ in a buffer composed of $10 \mathrm{mM}$ Tris- $\mathrm{HCl}$ pH 7.5, $200 \mathrm{mM} \mathrm{NaCl}, 0.6 \mathrm{mM}$ EDTA, $55 \mu \mathrm{g} \mathrm{ml}^{-1} \mathrm{BSA}, 10 \%$ glycerol, $0.2 \mathrm{mM}$ DTT. A circular-relaxed plasmid or linear DNA $(1.2 \mu \mathrm{g})$ was added to the reaction and incubated at $37^{\circ} \mathrm{C}$ for $2 \mathrm{~h}$. For chromatin assembly on circular-relaxed plasmid, topoisomerase I was added to the reaction and further incubated for $1 \mathrm{~h}$. Assembly was analysed by gel-shift, sucrose gradient sedimentation, DNA supercoiling assay and MNase digestion, as described below.

Chromatin purification by sucrose gradient sedimentation. Nucleosomes were reconstituted by histone chaperone-mediated chromatin assembly or salt dilution onto a linear 561-bp DNA (with three 147-bp '601' sequence separated by 60 bp of linker DNA), 207-bp CEN3, 5S or 601 DNA. About $100 \mu \mathrm{l}$ of the reconstituted chromatin samples (concentrated when needed) were loaded onto a $10 \mathrm{ml} \mathrm{5-25 \%}$ sucrose gradient (in $25 \mathrm{mM}$ Tris- $\mathrm{HCl} ; 7.5,1 \mathrm{mM}$ EDTA, $1 \mathrm{mM} \beta-\mathrm{Me}, 100 \mathrm{mM}$ $\mathrm{NaCl}$ ) and spun for $18 \mathrm{~h}$ at 28,000 r.p.m. at $4^{\circ} \mathrm{C}$ in a Beckman SW 41 Ti rotor (Beckman Coulter). The gradients were fractionated by taking out $200 \mu \mathrm{l}$ from the top and fractions were analysed by $5 \%$ native PAGE or $4-12 \%$ Bis-Tris SDS-PAGE (Bio-Rad).

DNA supercoiling assay. Nucleosomes were assembled onto a relaxed circular plasmid (pBR322 or pCR2.1-4 (CEN3 + CEN6)) using histone chaperone (yNap1, $\mathrm{Scm} 3$ or $\left.\mathrm{Scm} 3_{63-189}\right)$-mediated chromatin assembly approach, as described above. The plasmid pCR2.1-4(CEN3 + CEN6) contains four copies of CEN3 and CEN6 DNA (a gift from the Henikoff Laboratory). Briefly, histone octamer was preincubated with yNap1, $\mathrm{Scm} 3$ or $\mathrm{Scm} 3_{63-189}$. A relaxed circular plasmid was added and the reaction was incubated for $2 \mathrm{~h}$ at $37^{\circ} \mathrm{C}$. A volume of $8 \mathrm{U}$ wheat germ topoisomerase I (Promega) was added and the reaction was incubated at $37^{\circ} \mathrm{C}$ for $1 \mathrm{~h}$. The reaction was stopped and deproteinized by adding $0.5 \%$ SDS and $0.2 \mathrm{mg} \mathrm{ml}^{-1}$ proteinase $\mathrm{K}$ and incubating at $50^{\circ} \mathrm{C}$ for $30 \mathrm{~min}$. The DNA extracted was analysed for supercoiling on a $1 \%$ agarose gel. Negative and positive plasmid supercoiling was distinguished by running the agarose gel in presence of $1-2 \mu \mathrm{g} \mathrm{ml}^{-1}$ chloroquine ${ }^{42}$, and by two-dimensional gel analysis in which the samples were run without cholorquine followed by electrophoresis in the second dimension in the presence of $10 \mu \mathrm{g} \mathrm{ml}^{-1}$ chloroquine ${ }^{43}$.

Micrococcal nuclease mapping of nucleosomes. Cse4-nucleosomes and canonical nucleosomes were reconstituted onto a 147-bp 601 DNA by salt dilution. Alternatively, reconstitutions were done on a linearized pBR322 plasmid DNA by yNap1-mediated chromatin assembly protocol. The nucleosome samples (reconstituted onto $\sim 5 \mu \mathrm{g}$ DNA) were digested with MNase (4,000 gel units; NEB) for the indicated times (in Supplementary Fig. S3) and quenched by adding EDTA to final concentration of $50 \mathrm{mM}$. The samples were deproteinized as described above, separated on a $5 \%$ native PAGE.

Unzipping single DNA molecules in an optical trap. Both the forward and reverse nucleosomal DNA templates (Supplementary Table S1) were prepared using published methods ${ }^{34,44}$. Cse4- and Sc-nucleosomes were assembled from recombinant yeast histones using salt dialysis. Nucleosomal DNA templates were unzipped using methods similar to those previously described ${ }^{34,44}$ (Supplementary Fig. S4) under the following buffer condition: $10 \mathrm{mM}$ Tris- $\mathrm{HCl}$ ( $\mathrm{pH}$ 7.5), $1 \mathrm{mM}$ EDTA, $100 \mathrm{mM}$ $\mathrm{NaCl}, 1 \mathrm{mM}$ DTT, 3\% (v/v) glycerol, 0.02\% (v/v) Tween-20, $2 \mathrm{mg} \mathrm{ml}^{-1} \mathrm{BSA}$.

Size-exclusion chromatography-multiangle light scattering. A volume of 100-120 $\mu \mathrm{l}$ of 1-2 $\mathrm{mg} \mathrm{ml}^{-1}$ nucleosomes reconstituted on 147-bp 601 DNA were loaded onto a Sephadex 200 ( $24 \mathrm{ml}$, GE Healthcare) size-exclusion column at a flow rate of $0.3 \mathrm{ml} \mathrm{min}^{-1}$ using an ÄKTA purifier HPLC system (GE Healthcare). The column was connected to a Dawn Heleos II (Wyatt Technologies) multiangle light scattering instrument, followed by a REx refractive index detector (Wyatt Technologies). To determine the concentration of the nucleosomes a differential index of diffraction $(\mathrm{d} n / \mathrm{d} c)$ of 0.175 was used.

Small-angle X-ray scattering. SAXS data were collected at the advanced light source beamline 12.3.1 and at the Stanford Synchrotron Radiation Light Source (SSRL) BL4-2. Nucleosome solutions identical to those used in SEC-MALS were prepared at $1-8 \mathrm{mg} \mathrm{ml}^{-1}$ in buffer composed of $25 \mathrm{mM}$ Tris- $\mathrm{HCl} \mathrm{pH} \mathrm{7.5,1} \mathrm{mM}$ EDTA and $0.2 \mathrm{mM}$ tris (2-carboxyethyl) phosphine. Exposure series of 2, 10 and $2 \mathrm{~s}$ were performed at the advanced light source to rule out radiation damage. Data for the reference buffer were collected with the same time series for subsequent intensity-corrected background subtraction and radial averaging of all images. At SSRL, ten $1 \mathrm{~s}$ exposures were collected in different spots of a capillary containing the sample. The data were analysed by SasTool and the exposures, which were significantly different, were excluded. Exposures were averaged and buffer scattering was subtracted using SasTool.

Data were processed by PRIMUS ${ }^{45}$. Guinier, Porod and Kratky analyses were performed with the same programme. Maximum particle dimensions were determined by indirect Fourier transform with $\mathrm{GNOM}^{46}$. Particle reconstructions were performed with the program DAMMIN ${ }^{47}$. Ten reconstructions were averaged and superimposed ${ }^{48}$. Molecular envelopes were constructed with Situs ${ }^{49}$ and displayed with $\mathrm{VMD}^{50}$.

\section{References}

1. Cleveland, D. W., Mao, Y. \& Sullivan, K. F. Centromeres and kinetochores: from epigenetics to mitotic checkpoint signaling. Cell 112, 407-421 (2003).

2. Cooper, J. L. \& Henikoff, S. Adaptive evolution of the histone fold domain in centromeric histones. Mol. Biol. Evol. 21, 1712-1718 (2004).

3. Smith, C. M. et al. Heritable chromatin structure: mapping 'memory' in histones H3 and H4. Proc. Natl Acad. Sci. USA 26, 26 (2002).

4. Foltz, D. R. et al. The human CENP-A centromeric nucleosome-associated complex. Nat. Cell Biol. 8, 458-469 (2006)

5. Sekulic, N., Bassett, E. A., Rogers, D. J. \& Black, B. E. The structure of (CENPA-H4)(2) reveals physical features that mark centromeres. Nature 467, 347-351 (2010).

6. Yoda, K. et al. Human centromere protein A (CENP-A) can replace histone $\mathrm{H} 3$ in nucleosome reconstitution in vitro. Proc. Natl Acad. Sci. USA 97, 7266-7271 (2000).

7. Dalal, Y., Wang, H., Lindsay, S. \& Henikoff, S. Tetrameric structure of centromeric nucleosomes in interphase Drosophila cells. PLoS Biol. 5, e218 (2007).

8. Camahort, R. et al. Cse 4 is part of an octameric nucleosome in budding yeast. Mol. Cell 35, 794-805 (2009).

9. Chen, Y. et al. The $\mathrm{N}$ terminus of the centromere H3-like protein Cse4p performs an essential function distinct from that of the histone fold domain. Mol. Cell Biol. 20, 7037-7048 (2000).

10. Mizuguchi, G. et al. ATP-driven exchange of histone $\mathrm{H} 2 \mathrm{AZ}$ variant catalyzed by SWR1 chromatin remodeling complex. Science 303, 343-348 (2004).

11. Mizuguchi, G., Xiao, H., Wisniewski, J., Smith, M. M. \& Wu, C. Nonhistone $\mathrm{Scm} 3$ and histones CenH3-H4 assemble the core of centromere-specific nucleosomes. Cell 129, 1153-1164 (2007).

12. Williams, J. S., Hayashi, T., Yanagida, M. \& Russell, P. Fission yeast Scm3 mediates stable assembly of Cnp1/CENP-A into centromeric chromatin. $\mathrm{Mol}$. Cell 33, 287-298 (2009).

13. Furuyama, T. \& Henikoff, S. Centromeric nucleosomes induce positive DNA supercoils. Cell 138, 104-113 (2009).

14. Dechassa, M. L., D’Arcy, S. \& Luger, K. A positive spin on the centromere. Cell 138, 22-24 (2009).

15. Black, B. E. et al. Structural determinants for generating centromeric chromatin. Nature 430, 578-582 (2004).

16. Fitzgerald-Hayes, M., Clarke, L. \& Carbon, J. Nucleotide sequence comparisons and functional analysis of yeast centromere DNAs. Cell 29, 235-244 (1982).

17. Furuyama, S. \& Biggins, S. Centromere identity is specified by a single centromeric nucleosome in budding yeast. Proc. Natl Acad. Sci. USA 104, 14706-14711 (2007).

18. Niedenthal, R. K., Sen-Gupta, M., Wilmen, A. \& Hegemann, J. H. Cpf1 protein induced bending of yeast centromere DNA element I. Nucleic Acids Res. 21, 4726-4733 (1993).

19. Sears, D. D., Hegemann, J. H., Shero, J. H. \& Hieter, P. Cis-acting determinants affecting centromere function, sister-chromatid cohesion and reciprocal recombination during meiosis in Saccharomyces cerevisiae. Genetics 139, 1159-1173 (1995).

20. Ortiz, J., Stemmann, O., Rank, S. \& Lechner, J. A putative protein complex consisting of Ctf19, Mcm21, and Okp1 represents a missing link in the budding yeast kinetochore. Genes Dev. 13, 1140-1155 (1999).

21. Camahort, R. et al. $\mathrm{Scm} 3$ is essential to recruit the histone $\mathrm{h} 3$ variant cse 4 to centromeres and to maintain a functional kinetochore. Mol. Cell 26, 853-865 (2007).

22. Pidoux, A. L. et al. Fission yeast $\mathrm{Scm} 3$ : A CENP-A receptor required for integrity of subkinetochore chromatin. Mol. Cell 33, 299-311 (2009).

23. Dunleavy, E. M. et al. HJURP is a cell-cycle-dependent maintenance and deposition factor of CENP-A at centromeres. Cell 137, 485-497 (2009).

24. Foltz, D. R. et al. Centromere-specific assembly of CENP-a nucleosomes is mediated by HJURP. Cell 137, 472-484 (2009).

25. Shuaib, M., Ouararhni, K., Dimitrov, S. \& Hamiche, A. HJURP binds CENP-A via a highly conserved $\mathrm{N}$-terminal domain and mediates its deposition at centromeres. Proc. Natl Acad. Sci. USA 107, 1349-1354 (2010).

26. Aravind, L., Iyer, L. M. \& Wu, C. Domain architectures of the $\mathrm{Scm} 3 p$ protein provide insights into centromere function and evolution. Cell Cycle 6, 2511-2515 (2007)

27. Luger, K., Mader, A. W., Richmond, R. K., Sargent, D. F. \& Richmond, T. J. Crystal structure of the nucleosome core particle at 2.8 A resolution. Nature 389, 251-260 (1997).

28. Lowary, P. T. \& Widom, J. New DNA sequence rules for high affinity binding to histone octamer and sequence-directed nucleosome positioning. J. Mol. Biol. 276, 19-42 (1998). 
29. Furuyama, T., Dalal, Y. \& Henikoff, S. Chaperone-mediated assembly of centromeric chromatin in vitro. Proc. Natl Acad. Sci. USA 103, 6172-6177 (2006).

30. Davey, C. A., Sargent, D. F., Luger, K., Maeder, A. W. \& Richmond, T. J. Solvent mediated interactions in the structure of the nucleosome core particle at $1.9 \mathrm{a}$ resolution. J. Mol. Biol. 319, 1097-1113 (2002).

31. Mangenot, S., Leforestier, A., Vachette, P., Durand, D. \& Livolant, F. Saltinduced conformation and interaction changes of nucleosome core particles. Biophys. J. 82, 345-356 (2002).

32. Yang, C., van der Woerd, M. J., Muthurajan, U. M., Hansen, J. C. \& Luger, K. Biophysical analysis and small-angle X-ray scattering-derived structures of MeCP2-nucleosome complexes. Nucleic Acids Res. (e-pub ahead of print).

33. Kingston, I. J., Yung, J. S. \& Singleton, M. R. Biophysical characterization of the centromere-specific nucleosome from budding yeast. J. Biol. Chem. 286, 4021-4026 (2011)

34. Hall, M. A. et al. High-resolution dynamic mapping of histone-DNA interactions in a nucleosome. Nat. Struct. Mol. Biol. 16, 124-129 (2009).

35. Segal, E. \& Widom, J. From DNA sequence to transcriptional behaviour: a quantitative approach. Nat. Rev. Genet. 10, 443-456 (2009).

36. Stoler, S. et al. Scm3, an essential Saccharomyces cerevisiae centromere protein required for G2/M progression and Cse4 localization. Proc. Natl Acad. Sci. USA 104, 10571-10576 (2007).

37. Park, Y. J., McBryant, S. J. \& Luger, K. A beta-hairpin comprising the nuclear localization sequence sustains the self-associated states of Nucleosome Assembly Protein 1. J. Mol. Biol. 375, 1076-1085 (2008).

38. Visnapuu, M. L. \& Greene, E. C. Single-molecule imaging of DNA curtains reveals intrinsic energy landscapes for nucleosome deposition. Nat. Struct. Mol. Biol. 16, 1056-1062 (2009)

39. Dyer, P. N. et al. Reconstitution of nucleosome core particles from recombinant histones and DNA. Methods Enzymol. 375, 23-44 (2004).

40. Luger, K., Rechsteiner, T. J. \& Richmond, T. J. Preparation of nucleosome core particle from recombinant histones. Methods Enzymol. 304, 3-19 (1999).

41. Fujii Nakata, T., Ishimi, Y., Okuda, A. \& Kikuchi, A. Functional analysis of nucleosome assembly protein, NAP-1. The negatively charged $\mathrm{COOH}$-terminal region is not necessary for the intrinsic assembly activity. J. Biol. Chem. 267, 20980-20986 (1992).

42. Clark, D. J. \& Leblanc, B. Analysis of DNA supercoiling induced by DNAprotein interactions. Methods Mol. Biol. 543, 523-535 (2009).

43. Peterson, S., Danowit, R., Wunsch, A. \& Jackson, V. NAP1 catalyzes the formation of either positive or negative supercoils on DNA on basis of the dimer-tetramer equilibrium of histones H3/H4. Biochemistry 46, 8634-8646 (2007).

44. Shundrovsky, A., Smith, C. L., Lis, J. T., Peterson, C. L. \& Wang, M. D. Probing SWI/SNF remodeling of the nucleosome by unzipping single DNA molecules. Nat. Struct. Mol. Biol. 13, 549-554 (2006).

45. Konarev, P. V., Volkov, V. V., Sokolova, A. V., Koch, M. H. J. \& Svergun, D. I. PRIMUS: a Windows PC-based system for small-angle scattering data analysis. J. Appl. Crystallogr. 36, 1277-1282 (2003).
46. Svergun, D. I. Determination of the regularization parameter in indirecttransform methods using perceptual criteria. J. Appl. Crystallogr. 25, 495-503 (1992).

47. Svergun, D. I. Restoring low resolution structure of biological macromolecules from solution scattering using simulated annealing. Biophys. J. 76, 2879-2886 (1999).

48. Kozin, M. B. \& Svergun, D. I. Automated matching of high- and low-resolution structural models. J. Appl. Crystallogr. 34, 33-41 (2001).

49. Wriggers, W., Milligan, R. A. \& McCammon, J. A. Situs: a package for docking crystal structures into low-resolution maps from electron microscopy. J. Struct. Biol. 125, 185-195 (1999).

50. Humphrey, W., Dalke, A. \& Schulten, K. VMD: visual molecular dynamics. J. Mol. Graph 14, 33-38, 27-28 (1996).

51. Schneidman-Duhovny, D., Hammel, M. \& Sali, A. FoXS: a web server for rapid computation and fitting of SAXS profiles. Nucleic Acids Res. 38 (Suppl), W540-W544 (2010)

\section{Acknowledgments}

We thank Steven Harrison and Stephen Henikoff for plasmids; K. Brown, U. Muthurajan S. D'Arcy and D. Winkler for comments and discussion, and P. Dyer and A. White for help with reagents. This work was funded by 1P01GM088409-01A1 to K.L., NIHGM059849 to M.D.W., and by an AHA-10POST4190042 postdoctoral fellowship to M.L.D. M.D.W. and K.L. are supported by the Howard Hughes Medical Institute.

\section{Author contributions}

M.L.D. (with the help of K.W.) performed all experiments with the exception of those shown in Figure 3 and Supplementary Figures S4-S6, which were done by M.L., M.A.H. and M.D.W. M.L.D and K.L designed the experiments and wrote the manuscript, with contributions from M.D.W.

\section{Additional information}

Supplementary Information accompanies this paper at http://www.nature.com/ naturecommunications

Competing financial interests: The authors declare no competing financial interests.

Reprints and permission information is available online at http://npg.nature.com/ reprintsandpermissions/

How to cite this article: Dechassa, M. L. et al. Structure and Scm3-mediated assembly of budding yeast centromeric nucleosomes. Nat. Commun. 2:313 doi: 10.1038/ncomms1320 (2011).

License: This work is licensed under a Creative Commons Attribution-NonCommercialNoDerivative Works 3.0 Unported License. To view a copy of this license, visit http:// creativecommons.org/licenses/by-nc-nd/3.0/ 